\title{
Instabiity and Encapsulation of a Compound Liquid Jet*
}

\author{
Takao YOSHINAGA** and Michiko MAEDA*** \\ ** Graduate School of Engineering Science, Osaka University \\ Toyonaka, Osaka 560-8531, Japan \\ E-mail: yoshinag@me.es.osaka-u.ac.jp \\ *** Graduate School of Engineering Science, Osaka University \\ Toyonaka, Osaka 560-8531, Japan
}

\begin{abstract}
We analytically examine breakup phenomena of a compound liquid jet which consists of a gas or liquid core phase and a surrounding annular phase. Applying the long wave approximations to the basic equations and the boundary conditions for inviscid and incompressible fluids, simplified nonlinear equations are derived for large deformation of the jet. It is numerically shown for a doubly infinite jet that the core phase is periodically capsuled by the annular phase, whose profiles are largely affected by density ratios and velocity difference between the core and annular phases. On the other hand, for a semi-infinite jet emanating from a nozzle exit, breakup of the jet brings about encapsulation in the downstream, whose profile becomes more sensitive to input disturbances when the Weber number is small. For larger Weber number, however, the breakup profiles are almost the same as those in the doubly infinite jet. In order to see the validity of the present model, breakup profiles are also shown for the parameters used in the previous experiments.
\end{abstract}

Key words : Encapsulation, Compound Jet, Nonlinear, Surface Tension, Breakup

\section{Introduction}

A gas- or liquid-cored annular jet, which is called a compound liquid jet, is of great importance in various engineering and industrial applications such as coating, encapsulation and atomization techniques. ${ }^{(1),(2)}$

It has been experimentally shown that the jet breaks up by sealing-off and ballooning of the annular phase, which results in encapsulations of the core phase. Hertz and Hermanrud ${ }^{(3)}$ observed two different encapsulations depending upon the surface tension ratios between outer and inner interfaces for the same flow velocity of the core and annular phases. On the other hand, in a gas-cored liquid jet with different flow velocities between both phases, Kendall ${ }^{(4)}$ observed that an optimum velocity ratio of the gas to the annular exists for naturally producing spherical liquid shells.

In spite of such large deformations of the jet observed in the experiments, most of the analytical treatments are limited to small deformations, that is, the linear analysis ${ }^{(5)-(8)}$. For axisymmetric long wave disturbances, the analysis shows that two different unstable modes exist due to the surface tension instability. They are referred to as the para-sinuous (or stretching) and para-varicose (or squeezing) modes, which are reduced to the sinuous and varicose modes in a planar sheet in the limit of large annular radius. Besides, for rather short wave disturbances, an additional unstable mode appears due to the Kelvin-Helmholtz instability (K-H instability, for short) or the aerodynamic instability, where this mode merges with the para-sinuous mode for large velocity difference.

Since such instabilities bring about amplification of disturbances on the jet even when initial disturbances are sufficiently small, it is evident that the nonlinearity becomes important 
in a finite time. Furthermore, even for a linearly stable wave, the nonlinearity also becomes important when the initial amplitude is sufficiently large. In order to explain such nonlinear behavior as breakup and encapsulation, analytical studies based on the long wave approximation have been performed on liquid jets and sheets ${ }^{(9),(10)}$, as well as numerical analyses using the discrete vortex method ${ }^{(11),(12)}$ and others ${ }^{(13)}$. In particular, Lee and Wang ${ }^{(14)}$ derived two-dimensional nonlinear equations for an annular sheet without a core flow. Although their equations only allow the para-sinuous mode because of sufficiently thin sheet thickness, they explained the experimental results of Kendall ${ }^{(4)}$ by using the phenomenological model based on this mode. Later, Mehring and Sirignano ${ }^{(15),(16)}$ derived equations for finite thickness of the annular sheet by averaging the dependent variables over the sheet thickness, for which both para-sinuous and para-varicose modes are allowed. By using the equations they examined the temporal and spatial behavior of the annular sheet and clarify the breakup process. Yoshinaga and Kotani ${ }^{(17),(18)}$ showed that the same equations as Mehring and Sirignano can be also obtained from the straightforward extension of the analysis in Lee and Wang ${ }^{(14)}$ to the finite thickness. Furthermore, by using the averaging procedure, Sano and Funakoshi ${ }^{(19)}$ derived nonlinear equations for an annular sheet which consists of two immiscible liquid layers, where another unstable mode appears in addition to the usual para-sinuous and para-varicose modes.

As is seen in the above, in spite of the importance of the compound jet in many applications, most of the nonlinear analyses have exclusively dealt with column or annular sheet jets. In this paper, we analytically examine the breakup and encapsulation phenomena of an inviscid compound jet in which the core column phase interacts with the annular phase. For this end, we use the long wave approximation with different expansion parameters in the core and annular phases and analytically derive two-dimensional evolution equations for the compound jet. The organization of this paper is as follows: in the next section, we formulate our problem and derive the nonlinear equations by using the long wave approximations. In Section 3, we proceed to the numerical analysis to understand the breakup and encapsulation process. In order to see the validity of the present model, the analysis is also compared with the previous experiments $^{(3),(4)}$. Finally, our conclusions are presented in Section 4.

\section{Formulation}

Figure 1 shows the schematic of the jet in the $(r, z)$ axisymmetric coordinate system. Denoting the core and annular phases by the subscripts $j=1$ and 2 , respectively, the velocity components are denoted by $\left(v_{j}, u_{j}\right)$, densities by $\rho_{j}$ and pressures by $p_{j}$. The surfaces are given by $r=h_{j}$ and the surface tension of the interfaces by $\sigma_{j}$, while the pressure of the surrounding ambient gas $p_{3}$ is constant and its density is ignored. For convenience of the later analysis, the thickness of the annular jet $b\left(=h_{2}-h_{1}\right)$ and the radius of the mid-plane $R\left(=\left(h_{2}+h_{1}\right) / 2\right)$ are introduced. In the analysis, we assume that the core and annular jets are inviscid and incompressible, and the gravitational force is negligible.

The basic equations are given by the continuity and momentum equations for the core phase $\left(0<r<h_{1}\right)$ and for the annular phase $\left(h_{1}<r<h_{2}\right)$

$$
\begin{gathered}
\frac{\partial u_{j}}{\partial z}+\frac{1}{r} \frac{\partial\left(r v_{j}\right)}{\partial r}=0, \\
\frac{\partial u_{j}}{\partial t}+u_{j} \frac{\partial u_{j}}{\partial z}+v_{j} \frac{\partial u_{j}}{\partial r}=-\frac{1}{\rho_{j}} \frac{\partial p_{j}}{\partial z}, \\
\frac{\partial v_{j}}{\partial t}+u_{j} \frac{\partial v_{j}}{\partial z}+v_{j} \frac{\partial v_{j}}{\partial r}=-\frac{1}{\rho_{j}} \frac{\partial p_{j}}{\partial r} .
\end{gathered}
$$

On the other hand, the boundary conditions are given by the kinematic conditions on $r=h_{1}$

$$
\begin{aligned}
& v_{1}=\frac{\partial h_{1}}{\partial t}+u_{1} \frac{\partial h_{1}}{\partial z}, \\
& v_{2}=\frac{\partial h_{1}}{\partial t}+u_{2} \frac{\partial h_{1}}{\partial z},
\end{aligned}
$$


and on $r=h_{2}$

$$
v_{2}=\frac{\partial h_{2}}{\partial t}+u_{2} \frac{\partial h_{2}}{\partial z}
$$

while from the dynamical conditions on $r=h_{1}$ and $h_{2}$, respectively,

$$
\begin{aligned}
& p_{1}=p_{2}+\sigma_{1} \kappa_{1}, \\
& p_{2}=p_{3}+\sigma_{2} \kappa_{2},
\end{aligned}
$$

where the curvatures $\kappa_{j}$ are given as

$$
\kappa_{j}=h_{j}^{-1}\left[1+\left(\frac{\partial h_{j}}{\partial z}\right)^{2}\right]^{-1 / 2}-\left(\frac{\partial^{2} h_{j}}{\partial z^{2}}\right)\left[1+\left(\frac{\partial h_{j}}{\partial z}\right)^{2}\right]^{-3 / 2} .
$$

The basic equations and the boundary conditions can be simplified by using the long wave approximation in which sufficiently long waves are considered compared with the core radius and annular sheet thickness. In the present analysis, we introduce the approximations with different expansion parameters in the core and the annular phases. For the core $(j=1)$, we assume the variables $u_{1}$ and $p_{1}$ to be expanded in terms of $r^{2}$ of the core radius

$$
\begin{aligned}
& u_{1}=u_{1}^{(0)}+r^{2} u_{1}^{(2)}+\cdots, \\
& p_{1}=p_{1}^{(0)}+r^{2} p_{1}^{(2)}+\cdots .
\end{aligned}
$$

Substituting the above expansion into Eq.(1), we have the following equation in the lowest order of the approximation:

$$
v_{1}=-\frac{r}{2} \frac{\partial u_{1}^{(0)}}{\partial z}-\frac{r^{3}}{4} \frac{\partial u_{1}^{(2)}}{\partial z}-\cdots
$$

Using the above results into Eq. (2), we have the following equation in the lowest order of the approximation:

$$
\frac{\partial u_{1}^{(0)}}{\partial t}+u_{1}^{(0)} \frac{\partial u_{1}^{(0)}}{\partial z}=-\frac{1}{\rho_{1}} \frac{\partial p_{1}^{(0)}}{\partial z},
$$

Making use of (10) into the boundary condition (4), we have

$$
\frac{\partial h_{1}}{\partial t}+u_{1}^{(0)} \frac{\partial h_{1}}{\partial z}=-\frac{h_{1}}{2} \frac{\partial u_{1}^{(0)}}{\partial z},
$$

while the other condition (7) can be approximated to be

$$
p_{1}^{(0)}=\left.p_{1}\right|_{h_{1}}=\left.p_{2}\right|_{h_{1}}-\sigma_{1} \kappa_{1} .
$$

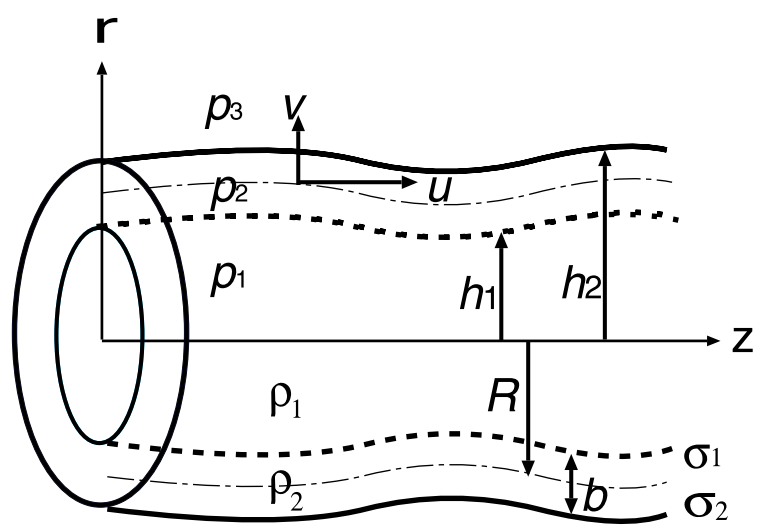

Fig. 1 Schematic of the compound liquid jet. 
Thus, the core phase is described by Eqs. (11) and (12) under the condition (13).

Next, for the annular phase $(j=2)$, we introduce the following power series expansion in terms of $(r-R)$ like the annular thickness:

$$
\begin{aligned}
& u_{2}=u_{2}^{(0)}+u_{2}^{(1)}(r-R)+u_{2}^{(2)}(r-R)^{2}+\cdots, \\
& v_{2}=v_{2}^{(0)}+v_{2}^{(1)}(r-R)+v_{2}^{(2)}(r-R)^{2}+\cdots \\
& p_{2}=p_{2}^{(0)}+p_{2}^{(1)}(r-R)+p_{2}^{(2)}(r-R)^{2}+\cdots
\end{aligned}
$$

Noting that $r^{-1}=R^{-1}[1+(r-R) / R]^{-1}$ and substituting the above representations into Eq. (1), we have in the lowest order of the approximation

$$
\frac{v_{2}^{(0)}}{R}+v_{2}^{(1)}+\frac{\partial u_{2}^{(0)}}{\partial z}-u_{2}^{(1)} \frac{\partial R}{\partial z}=0 .
$$

From the kinematic condition (5) on $r=h_{1}$ we have

$$
\left.v_{2}\right|_{h_{1}}=\frac{\partial h_{1}}{\partial t}+\left.u_{2}\right|_{h_{1}} \frac{\partial h_{1}}{\partial z}
$$

and from (6) on $r=h_{2}$

$$
\left.v_{2}\right|_{h_{2}}=\frac{\partial h_{2}}{\partial t}+\left.u_{2}\right|_{h_{2}} \frac{\partial h_{2}}{\partial z} .
$$

Taking account of $R=\left(h_{1}+h_{2}\right) / 2$ and $b=h_{2}-h_{1}$, and adding Eqs. (16) and (17) with the help of (14a) and (14b), we have the 'kinematic condition'

$$
v_{2}^{(0)}=\frac{\partial R}{\partial t}+u_{2}^{(0)} \frac{\partial R}{\partial z}
$$

while subtracting (16) from (17) with the help of (14a) and (14b), we have

$$
b\left(v_{2}^{(1)}-u_{2}^{(1)} \frac{\partial R}{\partial z}\right)=\frac{\partial b}{\partial t}+u_{2}^{(0)} \frac{\partial b}{\partial z} .
$$

Eliminating $v_{2}^{(1)}$ in (15) and (19), we have the 'continuity equation'

$$
\frac{\partial b}{\partial t}+\frac{\partial\left(b u_{2}^{(0)}\right)}{\partial z}+\frac{b v_{2}^{(0)}}{R}=0 .
$$

Using (18) into Eqs. (2) and (3), we have the 'momentum equations'

$$
\begin{aligned}
& \frac{\partial u_{2}^{(0)}}{\partial t}=-u_{2}^{(0)} \frac{\partial u_{2}^{(0)}}{\partial z}-\frac{1}{\rho_{2}}\left(\frac{\partial p_{2}^{(0)}}{\partial z}-p_{2}^{(1)} \frac{\partial R}{\partial z}\right), \\
& \frac{\partial v_{2}^{(0)}}{\partial t}=-u_{2}^{(0)} \frac{\partial v_{2}^{(0)}}{\partial z}-\frac{p_{2}^{(1)}}{\rho_{2}}
\end{aligned}
$$

Since

$$
\begin{aligned}
& \left.p_{2}\right|_{h_{1}}=p_{2}^{(0)}-\frac{b}{2} p_{2}^{(1)}+\cdots, \\
& \left.p_{2}\right|_{h_{2}}=p_{2}^{(0)}+\frac{b}{2} p_{2}^{(1)}+\cdots,
\end{aligned}
$$

from $(14 c)$, we can write

$$
\begin{aligned}
& p_{2}^{(0)}=\frac{1}{2}\left(\left.p_{2}\right|_{h_{1}}+\left.p_{2}\right|_{h_{2}}\right) \equiv P, \\
& p_{2}^{(1)}=\frac{1}{b}\left(\left.p_{2}\right|_{h_{2}}-\left.p_{2}\right|_{h_{1}}\right) \equiv \frac{\Delta P}{b} .
\end{aligned}
$$

On the other hand, since the boundary conditions (7) and (8) can be written as

$$
\begin{aligned}
& \left.p_{1}\right|_{h_{1}}=\left.p_{2}\right|_{h_{1}}+\sigma_{1} \kappa_{1}, \\
& \left.p_{2}\right|_{h_{2}}=p_{3}+\sigma_{2} \kappa_{2},
\end{aligned}
$$


we can obtain from Eqs. (24)

$$
\begin{aligned}
& P=\frac{1}{2}\left(\left.p_{1}\right|_{h_{1}}-\sigma_{1} \kappa_{1}\right)+\frac{1}{2}\left(p_{3}+\sigma_{2} \kappa_{2}\right), \\
& \Delta P=-\left(\left.p_{1}\right|_{h_{1}}-\sigma_{1} \kappa_{1}\right)+p_{3}+\sigma_{2} \kappa_{2} .
\end{aligned}
$$

As a result, Eqs. (21) and (22) can be written as

$$
\begin{aligned}
& \frac{\partial u_{2}^{(0)}}{\partial t}=-u_{2}^{(0)} \frac{\partial u_{2}^{(0)}}{\partial z}-\frac{1}{\rho_{2}}\left(\frac{\partial P}{\partial z}-\frac{\Delta P}{b} \frac{\partial R}{\partial z}\right), \\
& \frac{\partial v_{2}^{(0)}}{\partial t}=-u_{2}^{(0)} \frac{\partial v_{2}^{(0)}}{\partial z}-\frac{\Delta P}{\rho_{2} b} .
\end{aligned}
$$

Thus, the annular phase can be described by Eqs. (18), (20), (28) and (29) with the help of (26) and (27). In the derivation of these equations, we note that $O\left(\partial h_{1} / \partial z\right) \sim O\left(h_{1}\right)$ is assumed and higher order terms than $O\left(h_{1}\right)$ are neglected for the core, while $O(\partial b / \partial z) \sim O(b)$ is assumed and the terms higher than $O(b)$ are neglected for the annular sheet. As is seen from (13) and (23b), the boundary condition (7) on $r=h_{1}$ is valid in the present approximation as long as $O\left(h_{1}^{2}\right) \ll O(b)$.

Introducing the characteristic length $H$, velocity $U$ (these are to be specified later), time $H / U$ and pressure $\rho_{2} U^{2}$, Eqs. (11) and (12) for the core phase and Eqs. (18), (20), (28) and (29) for the annular phase are normalized as follows:

$$
\begin{aligned}
\frac{\partial h_{1}}{\partial t} & =-u_{1} \frac{\partial h_{1}}{\partial z}-\frac{h_{1}}{2} \frac{\partial u_{1}}{\partial z}, \\
\frac{\partial u_{1}}{\partial t} & =-u_{1} \frac{\partial u_{1}}{\partial z}-\frac{1}{\rho} \frac{\partial p_{1}}{\partial z}, \\
\frac{\partial R}{\partial t} & =v_{2}-u_{2} \frac{\partial R}{\partial z}, \\
\frac{\partial b}{\partial t} & =-\frac{\partial\left(b u_{2}\right)}{\partial z}-\frac{b v_{2}}{R}, \\
\frac{\partial u_{2}}{\partial t} & =-u_{2} \frac{\partial u_{2}}{\partial z}-\left(\frac{\partial P}{\partial z}-\frac{\Delta P}{b} \frac{\partial R}{\partial z}\right), \\
\frac{\partial v_{2}}{\partial t} & =-u_{2} \frac{\partial v_{2}}{\partial z}-\frac{\Delta P}{b},
\end{aligned}
$$

while we have from (26) and (27) with the help of (13)

$$
\begin{aligned}
P & =\frac{1}{2}\left(p_{1}+p_{3}\right)-\frac{1}{2 \mathrm{~Wb}}\left(\sigma \kappa_{1}-\kappa_{2}\right), \\
\Delta P & =-\left(p_{1}-p_{3}\right)+\frac{1}{\mathrm{~Wb}}\left(\sigma \kappa_{1}+\kappa_{2}\right),
\end{aligned}
$$

where the superscripts in $u_{1}^{(0)}, p_{1}^{(0)}, u_{2}^{(0)}$ and $v_{2}^{(0)}$ have been omitted. In the above representations, the density ratio $\rho=\rho_{1} / \rho_{2}$, the surface tension ratio $\sigma=\sigma_{1} / \sigma_{2}$ and the Weber number $\mathrm{Wb}=\rho_{2} H U^{2} / \sigma_{2}$ are introduced as the non-dimensional parameters.

Since $h_{1}=R-b / 2$, the above equations seem to be redundant with respect to $h_{1}, R$ and $b$, while $p_{1}$ is left to be undetermined. However, we can find that Eq. (30) yields the following second order differential equation of $p_{1}$ with respect to $z$ by using Eqs. (31) to (35) with the help of (36) and (37):

$$
A_{1} \frac{\partial^{2} p_{1}}{\partial z^{2}}+A_{2} \frac{\partial p_{1}}{\partial z}+A_{3} p_{1}+A_{4}=0
$$

where the coefficients $A_{1}$ to $A_{4}$ are functions of $R, b, u_{1}, u_{2}, v_{2}$, and their representations have been omitted. Consequently, the problem can be reduced to solving Eqs. (31) to (35) together with Eq. (38) with the help of (36) and (37). 


\section{Numerical Results}

We first consider a steady equilibrium state of an infinitely long jet, which is given by uniform radii $h_{1}=\bar{h}_{1}$ and $h_{2}=\bar{h}_{2}$, so that, $R=\bar{R}$ and $b=\bar{b}$, while constant axial velocities $u_{1}=\bar{u}_{1}, u_{2}=\bar{u}_{2}$ as well as vanishing radial velocities $v_{1}=v_{2}=0$. Since $\Delta P=0$ from (35) for $v_{2}=0$, we find that Eq. (37) yields

$$
\bar{p}_{1}=p_{3}+\frac{1}{\mathrm{~Wb}}\left(\frac{\sigma}{\bar{R}-\bar{b} / 2}+\frac{1}{\bar{R}+\bar{b} / 2}\right),
$$

where we have set $p_{1}=\bar{p}_{1}$ for the equilibrium. This relation implies that the core pressure $\bar{p}_{1}$ is always larger than the surrounding pressure $p_{3}$ due to the surface tension.

Now, we are going to numerically examine an initial value problem and an initialboundary value problem for disturbances superimposed on the above steady state. Numerical calculations are carried out as follows: Using the solutions $R, b, u_{1}, u_{2}, v_{2}$ and $p$ at a time $t$, we obtain the solutions except for $p$ at a next time $t+\Delta t$ with a time step $\Delta t$ by solving Eq. (31) to (35). By using these solutins at $t+\Delta t$, we can calculate $p$ at $t+\Delta t$ from Eq. (38). Such a procedure is iterated in each time step to have the evolution of the solutions. In the calculations, the 4th-order Runge-Kutta method is used for the time derivative, while the finite difference method is used for the spatial derivatives, where the temporal difference $\Delta t$ is taken to be $10^{-3}$ and the spatial difference $\Delta z$ to be 0.1 in most of the calculations.

\subsection{Temporal evolution}

We first examine the temporal evolutions for spatially periodic disturbances in a doubly infinite jet $(-\infty<z<\infty)$. For numerical accuracy, the volumes of the annular phase $V_{L}=$ $\int_{0}^{L} 2 \pi R b \mathrm{~d} z$ and the core phase $V_{C}=\int_{0}^{L}(r-b / 2)^{2} \mathrm{~d} z$ are, respectively, retained within $0.1 \%$ and $0.2 \%$ in relative errors for all time, when a spatial period is given by $L=2 \pi / k$ for a given wave number $k$ of an initial disturbance. The calculations are carried out for the initial condition given by $R=0.95+\eta \cos k z$ with the amplitude $\eta$ and its relevant linear solutions $u_{1}, u_{2}, v_{2}, b$, while they are terminated when the jet breaks up resulting from sufficiently thin annular thickness or small core radius. The parameters are taken to be $\eta=0.1, k=0.5$ $(L \equiv 2 \pi / k=4 \pi)$ and $\bar{b}=0.1$ or $\mathrm{Wb}=20$ since $\mathrm{Wb}=2 / \bar{b}$ when we choose $H=\bar{h}_{2}$ and $U=\sqrt{2 \sigma_{2} / \rho_{2} \bar{b}}$ (capillary velocity).

We show in Fig. 2 the initial profile of the jet and the periodic capsule profiles of the core when $\rho=0.001$ and $\bar{u}_{2}=0$. We note that the ordinate and abscissa are, respectively, denoted by $r$ and $z$ in all the figures here and hereafter. The initial deformation in Fig. 2(a) is developed as in Fig. 2(b) when $\bar{u}_{1}=\bar{u}_{2}=0$, while it develops as in Fig. 2(c) when $\bar{u}_{1}=5$ and $\bar{u}_{2}=0$. We find from these figures that the breakup is caused by encapsulation of the core phase by the annular phase, where the breakup time decreases as the flow velocity difference $\left|\bar{u}_{1}-\bar{u}_{2}\right|$ increases. However, the location of closing of the annular phase is found to shift downward when the velocity of the core is larger than the annular $\bar{u}_{1}>\bar{u}_{2}$ as in Fig. 2(c).

(a)

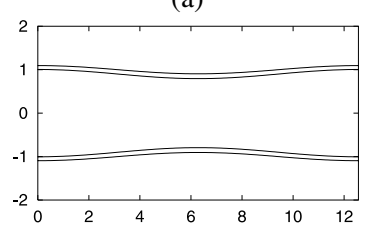

(b)

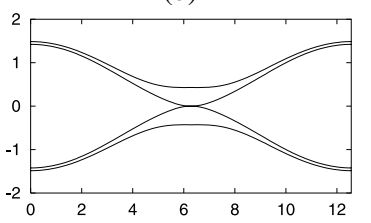

(c)

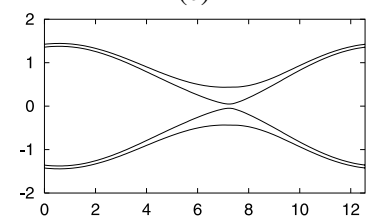

Fig. 2 Periodic capsule profiles for different $\bar{u}_{1}$ and $\bar{u}_{2}$ when $\rho=0.001, \sigma=1$ and $\bar{b}=0.1$; (a) initial profile at $t=0$, (b) $\bar{u}_{1}=\bar{u}_{2}=0$ at $t=2.05$ and (c) $\bar{u}_{1}=5$ and $\bar{u}_{2}=0$ at $t=1.55$.

We show in Fig. 3 the capsule profiles for larger density ratio $\rho=0.9$. From Fig. 3(a) for $\bar{u}_{1}=\bar{u}_{2}=0$, the annular phase is found to be more flattened in the trough and more blowing up than Fig. 2(b) for $\rho=0.001$. This may result from larger inertia of the core fluid, 
from which we can expect that capsules with two different sizes are simultaneously produced by closing at both ends of the flattened trough of the annular phase. Although the breakup time decreases as the velocity difference increases, it is found for larger density ratio that the velocity difference has much more influence on the breakup profile as in Fig. 3(b) for $\bar{u}_{1}=1$ and $\bar{u}_{2}=0$, where a wavy profile with short wave length appears due to the K-H instability.

(a)

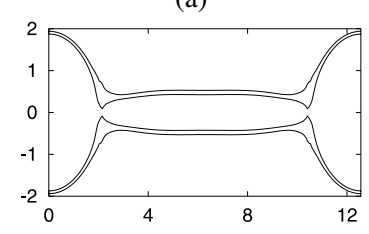

(b)

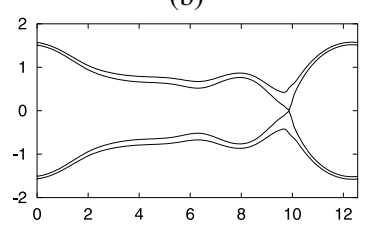

Fig. 3 Periodic capsule profiles for different $\bar{u}_{1}$ and $\bar{u}_{2}$ when $\rho=0.9, \sigma=1$ and $\bar{b}=0.1$; (a) $\bar{u}_{1}=\bar{u}_{2}=0$ at $t=20.18$ and (b) $\bar{u}_{1}=1$ and $\bar{u}_{2}=0$ at $t=12.9$.

\subsection{Spatial and temporal evolution}

In order to examine the spatial-temporal evolutions of disturbances on the jet, we next consider the initial-boundary value problem in a semi-infinite jet for $0 \leq z<\infty$, which is given by $v_{2}=\eta \sin \omega t$ and $p_{1}=\bar{p}_{1}+\bar{b} \omega \eta \cos \omega t$ with amplitude $\eta$ for $t \geq 0$ at the nozzle exit $z=0$. Numerical calculations are carried out in the spatial domain $0 \leq z \leq L=150$ and terminated when the jet breaks up otherwise a wave front of the disturbance reaches at the end of the domain. We require for the numerical accuracy in the relative errors that $V_{L}$ is less than $0.2 \%$ and $V_{C}$ less than $5 \%$. We take $\eta=0.1$ or -0.1 whose signs correspond to the initial directions of radial motion and we choose $H=\bar{h}_{2}$ and $U=\bar{u}_{2}$ as the parameters.

(a)

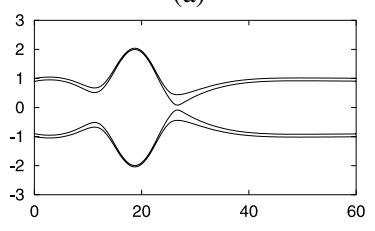

(b)

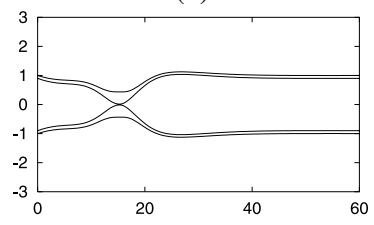

Fig. 4 Breakup profiles of the jet for different $\eta$ when $\rho=0.001, \sigma=1, \bar{b}=0.1$ and $\bar{u}_{1}=\bar{u}_{2}=1$, where $\mathrm{Wb}=125$ and $\omega=0.04$; (a) $\eta=0.1$ at $t=19.63$ and (b) $\eta=-0.1$ at $t=16.53$.

We first consider the case when Wb is small. In Fig. 4, the breakup profiles of the jet are shown for different $\eta$ when $\mathrm{Wb}=125$, where $\rho=0.001, \bar{u}_{1}=\bar{u}_{2}=1$ and $\omega=0.04$ are taken. In Fig. 4(a) for $\eta=0.1$, we find that the annular phase blows up like a balloon and breaks up at its right side (downstream) by closing the annular phase, while in Fig. 4(b) for $\eta=-0.1$ the jet breaks up only by closing the annular phase without blowing up. Therefore, the breakup profiles strongly depend upon the signs of $\eta$ of the disturbance at $z=0$.

In order to see effects of the flow velocity difference between the core and the annular, Fig. 5(a) shows the breakup profiles for $\bar{u}_{1}=0.6$, while Fig. 5(b) for 1.4 , when $\eta=0.1$, $\bar{u}_{2}=1, \rho=0.001$ and $\omega=0.04$. It can be seen from the figures that the location of closing in the annular phase moves to the right side (downstream) of the ballooning when $\bar{u}_{1}<\bar{u}_{2}$, while to the left side (upstream) when $\bar{u}_{1}>\bar{u}_{2}$. However, for $\eta=-0.1$, we cannot find any noticeable discrepancies on the breakup profiles between $\bar{u}_{1}=0.6$ in Fig. 6(a) and 1.4 in Fig. 6(b), where the jet breaks without blowing up and the breakup time slightly increases as $\bar{u}_{1}$ increases. As a result, we can say that the breakup profiles of the jet are sensitive to the input wave profiles rather than the velocity difference as long as $\mathrm{Wb}$ is small.

Next we consider when $\mathrm{Wb}$ is large. In Fig. 7, breakup profiles of the jet are shown for $\mathrm{Wb}=1280$, where $\bar{u}_{1}=\bar{u}_{2}=1, \rho=0.001$ and $\omega=0.0125$. In both Fig. 7(a) for $\eta=0.1$ and Fig. 7(b) for $\eta=-0.1$, we can see that the disturbance propagates downstream with blowing 
(a)

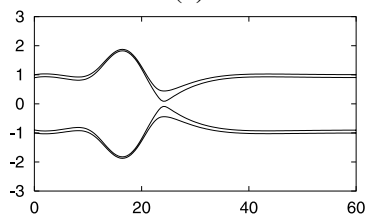

(b)

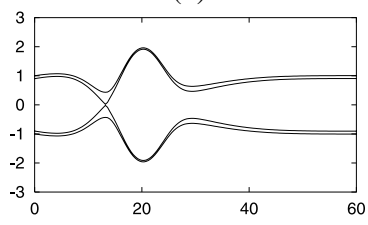

Fig. 5 Breakup profiles of the jet for different $\bar{u}_{1}$ when $\eta=0.1, \rho=0.001, \sigma=1$, $\bar{b}=0.1$ and $\bar{u}_{2}=1$, where $\mathrm{Wb}=125$ and $\omega=0.04$; (a) $\bar{u}_{1}=0.6$ at $t=18.225$ and (b) $\bar{u}_{1}=1.4$ at $t=20.0$.

(a)

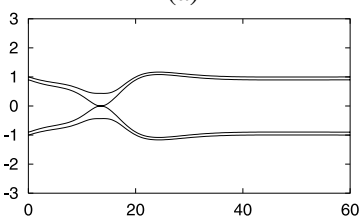

(b)

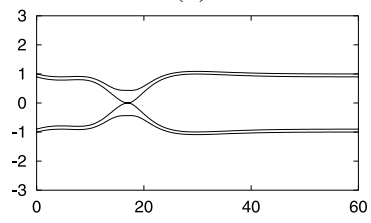

Fig. 6 Breakup profiles of the jet for different $\bar{u}_{1}$ when $\eta=-0.1, \sigma=1, \bar{b}=0.1$, $\rho=0.001$ and $\bar{u}_{2}=1$, where $\mathrm{Wb}=125$ and $\omega=0.04$; (a) $\bar{u}_{1}=0.6$ at $t=15.85$ and (b) $\bar{u}_{1}=1.4$ at $t=17.14$.

up as the time increases and breaks up by closing the annular phase. In spite of the difference of signs in $\eta$, we can find that the breakup profiles are close with each other except for the location of closing the annular phase, which is in contrast to Fig. 4 for which the breakup profiles strongly depend upon $\eta$. As is seen from the comparison between Figs. 7 and 4, the disturbance grows in a small distance of propagation for small $\mathrm{Wb}$, while it grows with propagation for a long distance in the downstream for large $\mathrm{Wb}$.

(a)

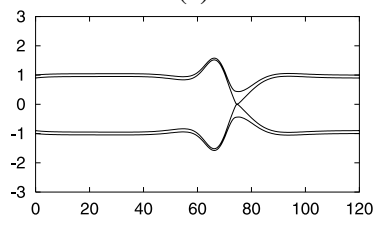

(b)

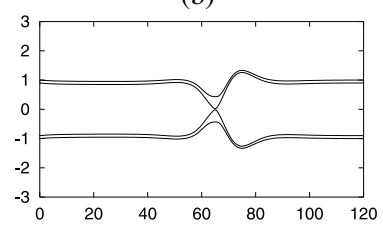

Fig. 7 Breakup profiles of the jet for different $\eta$ when $\rho=0.001, \sigma=1, \bar{b}=0.1$ and $\bar{u}_{1}=\bar{u}_{2}=1$, where $\mathrm{Wb}=1280$ and $\omega=0.0125$; (a) $\eta=0.1$ and (b) $\eta=-0.1$

We finally remark in Fig. 8 that other types of spatial-temporal evolutions can be seen for the jet with larger density ratio $\rho=0.9$ when $\eta=0.1, \bar{u}_{2}=1$ and $\bar{b}=0.1$. We find in Fig. 8(a) for $\mathrm{Wb}=5, \bar{u}_{1}=1$ and $\omega=1.6$ that the wavy disturbance with wavenumber $k \sim 1.1$ grows near $z=30$, which leads to the breakup by closing the annular phase, while in Fig. 8(b) for $\bar{u}_{1}=0, \mathrm{~Wb}=24.2$ and $\omega=0.727$ that the jet breaks through disintegration of the annular phase.

\subsection{Comparison with the previous experiments}

In order to see the validity of the present model, the encapsulation or breakup profiles are compared with those in the previous experiments. We first show in Fig. 9 the numerical results calculated for the parameters used in the experiment of Hertz and Hermanrud ${ }^{(3)}$. The experiment was performed for two cases of the parameters; in the first case the core phase is formed by $80 \% \mathrm{H}_{2} \mathrm{O}+20 \%$ glycerol+dye, flow rate $3.6 \times 10^{-5} \mathrm{~m}^{3} / \mathrm{s}$, radius $6.6 \times 10^{-5} \mathrm{~m}$ and density $1052 \mathrm{Kg} / \mathrm{m}^{3}$, while the annular phase by $80 \% \mathrm{H}_{2} \mathrm{O}+20 \%$ glycerol without dye, flow rate $6.4 \times 10^{-5} \mathrm{~m}^{3} / \mathrm{s}$, thickness $4.5 \times 10^{-5} \mathrm{~m}$ and density $1052 \mathrm{Kg} / \mathrm{m}^{3}$. In this case, the surface tension on the inner interface vanishes because of the almost same property in both liquids. Then, the flow parameters in the non-dimensional forms are read as $\mathrm{Wb}=48, \sigma=0, \rho=1$, 
(a)

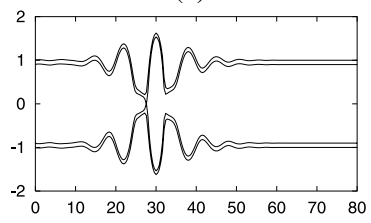

(b)

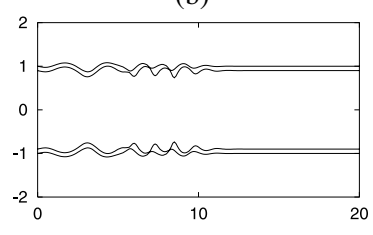

Fig. 8 Breakup profiles for $\rho=0.9$, where $\bar{u}_{2}=1, \eta=0.1, \sigma=1$ and $\bar{b}=0.1$; (a) $\bar{u}_{1}=1, \mathrm{~Wb}=5$ and $\omega=1.6$, while (b) $\bar{u}_{1}=0, \mathrm{~Wb}=24.2$ and $\omega=0.727$.

$\bar{u}_{1}=\bar{u}_{2}=1$ and $\bar{b}=0.3$. Figure 9(a) shows the calculated breakup profiles at $t=62.5$ when the initial-boundary condition is given by $u_{1}=1+0.005 \sin 0.79 t$ for $t \geq 0$ at $z=0$, where the forcing frequency is given from the observation. It is seen from this figure that both core radius and annular thickness of the jet vanish at $z \sim 55$. Although our calculation is terminated at this stage, we can expect that the jet will be disintegrated by simultaneous pinching of both phases. Such a breakup profile appears when the inner surface tension is sufficiently small and is something like that of a single phase jet. The experimental observation shows that the above breakup process is repeated periodically and encapsulated core liquid drops by the annular liquid are successively produced.

In the second case, however, the core jet is formed by $80 \% \mathrm{H}_{2} \mathrm{O}+20 \%$ glycerol+dye with flow rate $3.6 \times 10^{-5} \mathrm{~m}^{3} / \mathrm{s}$, radius $6.6 \times 10^{-5} \mathrm{~m}$ and density $1052 \mathrm{Kg} / \mathrm{m}^{3}$, while the annular jet by only silicon with flow rate $11.5 \times 10^{-5} \mathrm{~m}^{3} / \mathrm{s}$, thickness $7.0 \times 10^{-5} \mathrm{~m}$ and density $1052 \mathrm{Kg} / \mathrm{m}^{3}$. In this case, the surface tension on the inner interface is given by $5.2 \times 10^{-2} \mathrm{~N} / \mathrm{m}$ as the difference of those in both liquids. The normalized flow parameters are then read as $\mathrm{Wb}=48$, $\sigma=2.6, \rho=1, \bar{u}_{1}=\bar{u}_{2}=1, \bar{b}=0.5$. We show in Fig. 9(b) the breakup profiles at $t=26.5$ for the above parameters when the initial-boundary condition is given by $u_{1}=1-0.1 \sin 1.47 t$ for $t \geq 0$ at $z=0$. In contrast to Fig.9(a), the breakup appears at $z \sim 25$ only in the core phase with a vanishing radius because of much larger inner surface tension than the outer one. Although our calculation is also terminated at this stage, we can expect that the core phase will be disintegrated after this pinching, while the annular phase is not affected and left unchanged. This breakup profile is seen for the jet when the inner surface tension is much large and, so that, much different from a single phase jet. In the experiment, it is shown that such a breakup process is repeated periodically and a train of core liquid drops are observed in a sheath of the annular phase. Anyway, both numerical breakup profiles in Fig. 9 show that there are two typical basic process of the breakup, strongly depending upon the surface tension ratio.

(a)

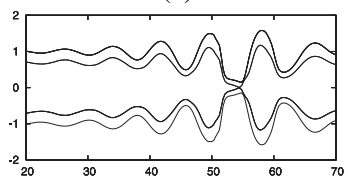

(b)

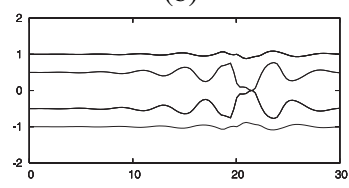

Fig. 9 Encapsulation of the core phase for the parameters in the experiment of Hertz and Hermanrud ${ }^{(3)}$, where almost the same liquid in the core and annular phases is used in (a), while different liquids are used in (b); (a) $\mathrm{Wb}=48, \sigma=0, \rho=1$, $\bar{u}_{1}=\bar{u}_{2}=1$ and $\bar{b}=0.3$ at $t=62.50$, (b) $\mathrm{Wb}=48, \sigma=2.6, \rho=1, \bar{u}_{1}=\bar{u}_{2}=1$, $\bar{b}=0.5$ at $t=26.5$.

Finally, we show in Fig.10 the results for the parameters in the experiment of Kendall ${ }^{(4)}$, whose experiment was performed in a gas-cored liquid jet with large velocity difference between the core and annular phases. The experiment was performed for different three core jet velocities when the other parameters are left unchanged. He estimated that an optimum velocity ratio of the core to the annular are given as 4.2 for natural producing of liquid shells. In his experiment, the three different core velocities are taken to be $1.52 \mathrm{~m} / \mathrm{s}, 4.58 \mathrm{~m} / \mathrm{s}$ and $13.73 \mathrm{~m} / \mathrm{s}$ for the annular jet velocity $1.09 \mathrm{~m} / \mathrm{s}$, when the core radius $1.25 \times 10^{-3} \mathrm{~m}$, the annu- 
lar thickness $0.75 \times 10^{-3} \mathrm{~m}$, the core density $1 \mathrm{~kg} / \mathrm{m}^{3}$ and the annular density $1000 \mathrm{Kg} / \mathrm{m}^{3}$. The parameters are then normalized as $\mathrm{Wb}=32.5, \sigma=1, \rho=0.001, \bar{u}_{2}=1$ and $\bar{b}=0.375$. When $R=0.813-0.005 \sin 2 t$ is given as the initial boundary condition, Figs. 10 (a), (b) and (c) show, respectively, the numerical profiles of the breakup for $\bar{u}_{1}=1.4,4.2$ and 12.6. In these figures, the annular phase is not perfectly closed because of the numerical resolution, since the closing speed near the breakup increases rapidly as $\bar{u}_{1}$ increases. However, we can find that the breakup time or length form the nozzle exit $z=0$ is reduced as $\bar{u}_{1}$ increases. In the experiment, pinching and disintegration of the jet appear after the above breakup to produce a gas-cored liquid shell. This process is repeated periodically and the period time or length between the produced shells becomes shorter and shorter as $\bar{u}_{1}$ increases. It is observed that the produced liquid shells are well separated with each other only for a suitable period length, which is determined by an optimum core flow velocity. Though we cannot find the optimum velocity from the numerical calculations, it is found, at least, that the breakup length strongly depends upon the core flow velocity, which allow us to expect that the shell formation period is proportional to the breakup length in our calculations.

(a)

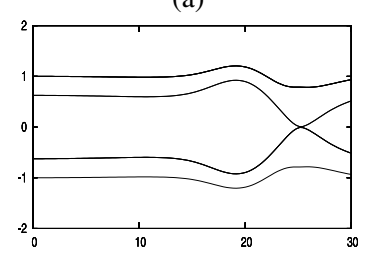

(b)

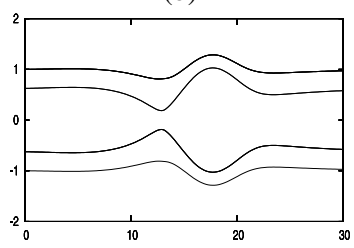

(c)

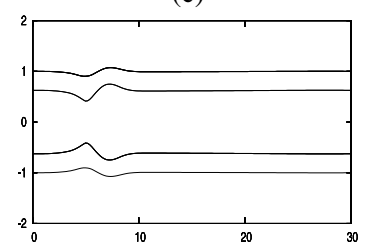

Fig. 10 Breakup profiles of the jet for the parameters in a gas-cored liquid jet experiment of Kendall ${ }^{(4)}$, where $\mathrm{Wb}=32.5, \sigma=1, \rho=0.001, \bar{u}_{2}=1$ and $\bar{b}=0.375$; (a) $\bar{u}_{1}=1.4$ at $t=22.55$, (b) $\bar{u}_{1}=4.2$ at $t=18.60$, (c) $\bar{u}_{1}=12.6$ at $t=8.059$.

\section{Conclusions}

We have analytically examined the encapsulation of the compound jet through the instability and breakup process. Using the long wave approximation with different expansion parameters to the core and annular jets, we derive the nonlinear evolution equations for large deformation.

The numerical analysis of the equations is performed to examine the encapsulation as a result of the nonlinear instability and breakup of the jet. In the doubly-infinite jet for periodic initial disturbances, the core phase is periodically capsuled by the annular phase, whose profiles are largely affected by the density ratio $\rho$ and velocity difference. In particular, each capsuled profile deviates from the symmetric form as the increase of the velocity difference, whose property is more enhanced for larger $\rho$.

On the other hand, for disturbances given at the nozzle exit in the semi-infinite jet, it is expected that the breakup of the jet appearing in the downward leads to the encapsulation of the core liquid. In particular, the breakup profiles strongly depends upon the boundary values at the nozzle exit for small $\mathrm{Wb}$, while their profiles are almost the same except for the location of closing the annular phase for larger $\mathrm{Wb}$. This may be closely related to the absolute instability for smaller $\mathrm{Wb}$ and convective instability for larger $\mathrm{Wb}$, which is known in the linear theory ${ }^{(2)}$. When $\mathrm{Wb}$ is sufficiently large, the effect of the boundary values at $z=0$ is less important and the breakup process is similar to that of the temporal instability in the doubly infinite jet. Whereas the jet breaks up by closing the annular phase in most of the cases, disintegration of the annular sheet is also observed as a particular case of the breakup profiles.

In spite of the inviscid present model, the numerical results calculated for the parameters used in the previous experiments can simulate, at least, qualitatively the experimental observa- 
tions in the breakup or encapsulation, where there are two typical breakup profiles depending upon the surface tension ratio. Because of the free boundary problem, we note that the viscosity may not seriously affect the behavior of the breakup of the jet, where Reynolds number is estimated to be several hundreds in both experiments.

\section{Acknowledgments}

This work has been partially supported by the Grant-in-Aid for Science Research from the Ministry of Education, Culture, Sports, Science and Technology of Japan (No.19560173).

\section{References}

( 1 ) Lefebvre, A.H., Atomization and sprays (Hemisphere, New York, 1989).

( 2 ) Lin, S. P., Breakup of liquid sheets and jets (Cambridge, 2003).

( 3 ) Hertz, C. H. and Hermanrud, B., "A liquid compound jet," J.FLuid Mech 131, 271 (1983).

( 4 ) Kendall, J. M., "Experiments on annular liquid jet instability and on the formation fo liquid shells," Phys. Fluids, 29, 2086 (1986).

( 5 ) Radev,S.P. and Gospodinov, P., "Numerical treatment of the steady flow on a liquid compound jet,” Int. J. Multiphase Flow 12, 997 (1986).

( 6 ) Panchagnula, M. V., Sojka, P. E. and Santangelo, J., "On the three-dimansional instability of a swirling, annular, inviscid liquid sheet subject to unequal gas velocity," Phys. Fluids 8, 3300 (1996).

( 7 ) Shen, J. and Li, X., "Instability of an annular viscous liquid jet," Acta Mechanica, 114, 167 (1996).

( 8 ) Chauhan, A. , Maldarelli, C. , Rumschitzki, D. S. and Papageorgiou, D. T., "Temporal and spatial instability of an inviscid compound jet," Rehol Acta, 35, 567 (1996).

( 9 ) Eggers, J., "Nonlinear dynamicsand breakup of free-surface flows," Rev. Mod. Phys. 69, 865 (1997).

(10) Mehring, C. and Sirignano, W. A., "Nonlinear capillary wave distortion and disintegration of thin planar liquid sheets," J. FLuid Mech. 388, 69 (1999).

(11) Rangel, R. H. and Sirignano, W. A., "The linear and nonlinear shear instability of a fluid sheet," Phys. Fluids A3, 2392 (1991).

(12) Kan, K. and Yoshinaga, T., "Instability of a planar liquid sheet with surrounding fluids between two parallelwalls," Fluid Dynamics Research 39, 389 (2007).

(13) Tchavdarov, B.M.,Minev, P.D., Radev, S.P., Comput. Methods Appl. Mech. Engrg. 118, 121 (1994).

(14) Lee, C. P. and Wang, T. G., "A theoretical model for the annular jet instability," Phys. Fluids 29, 2076 (1986).

(15) Mehring, C. and Sirignano, W. A., "Axisymmetric capillary waves on thin annular liquid sheets. I. Temporal stability," Phys. Fluids 12, 1417 (2000).

(16) Mehring, C. and Sirignano, W. A., "Axisymmetric capillary waves on thin annular liquid sheets. II. Spatial development," Phys. Fluids 12, 1440 (2000).

(17) Yoshinaga, T. and Kotani, K., "Modified membrane approximation on a thin liquid sheet," J. Phys. Soc. Japan, 70, 372 (2001).

(18) Yoshinaga, T. and Kotani, K., "Nonlinear behavior of an annular liquid sheet," in Proceedings 16th International Symposium in Nonlinear Acoustics, Moscow, edited by Rudenko, O. V. and Sapozhnikov, O. A., pp. 207-210.

(19) Sano, M. and Funakoshi, M., "Linear temporal instability of a two-layer axisymetric cylindrical liquid sheet,” J. Phys. Soc. Japan, 77, 044401 (2008). 